Title : will be set by the publisher

Editors : will be set by the publisher

EAS Publications Series, Vol. ?, 2018

\title{
THE SOLAR SIBLINGS IN THE GAIA ERA
}

\author{
C.A Martínez-Barbosa ${ }^{1}$, A.G.A. Brown ${ }^{1}$ and S. Portegies Zwart ${ }^{1}$
}

\begin{abstract}
We perform realistic simulations of the Sun's birth cluster in order to predict the current distribution of solar siblings in the Galaxy. We study the possibility of finding the solar siblings in the Gaia catalogue by using only positional and kinematic information. We find that the number of solar siblings predicted to be observed by Gaia will be around 100 in the most optimistic case, and that a phase space only search in the Gaia catalogue will be extremely difficult. It is therefore mandatory to combine the chemical tagging technique with phase space selection criteria in order to have any hope of finding the solar siblings.
\end{abstract}

\section{Introduction}

It is commonly accepted that disk stars are born in open clusters (Lada et al. 1993). Particular features of the solar system suggest the Sun was born in a cluster. For instance, the radioactive elements found in the meteorite fossil record (Looney et al. 2006) can be explained by a supernova explosion in the vicinity of the newborn Sun. The coplanarity of the Solar system planets and the eccentric orbits of the Kuiper-belt objects suggest that the Sun had a close encounter with another star belonging to the same open cluster (Morbidelli \& Levinson 2004). Such an encounter is expected to have occurred in the early history of the solar system (Malhotra 2008).

If the Sun was born in an open cluster 4.6 Gyr ago, the solar siblings -stars born in the same cluster- would be spread out over the Galactic disk. The identification of at least a small fraction of these stars would enable us to understand the conditions in which the solar system was formed.

In this paper we study the evolution and disruption of the Sun's birth cluster in the Galaxy by means of state-of-the-art simulations, taking into account the effects of the spiral arms and bar of the Galaxy as well as the internal processes

\footnotetext{
${ }^{1}$ Leiden Observatory, Leiden University, P.O. Box 9513 Leiden, 2300 RA, the Netherlands; e-mail: cmartinez@strw.leidenuniv.nl
}

(c) EDP Sciences 2018 DOI: (will be inserted later) 
Title : will be set by the publisher

in the cluster. We aim to predict the current phase-space distribution of the Solar siblings and the probability of detecting them in the large Gaia database.

\section{Simulations set up}

The Milky Way is modelled as an analytical potential composed by an axisymmetric component together with a rotating central bar and spiral arms. We use the potential of Allén \& Santillán (1991) to model the axisymmetric component of the Galaxy. The central bar is modelled with a Ferrers potential (Ferrers 1877) and the spiral arms are modeled as perturbations of the axisymmetric potential (Antoja et al. 2011). The bar and spiral arms are non transient structures that rotate as rigid bodies with different pattern speeds. The Galactic parameters used in the simulations are taken from Martínez-Barbosa et al. (2015).

The Sun's birth cluster on the other hand is modelled as a spherical distribution of stars that obey a Plummer potential (Plummer 1911). We use a Kroupa initial mass function (IMF) (Kroupa 2001) to model the mass distribution of the Sun's birth cluster. The minimum and maximum stellar masses are $0.08 M_{\odot}$ and $100 M_{\odot}$ respectively. Additionally, the metallicity of the Sun's birth cluster is assumed to be $Z=0.02$. Following Portegies Zwart (2009), we set the initial mass of the Sun's birth cluster between 500 and $3000 M_{\odot}$. The initial radius is set between 0.5 and 3 parsec.

The initial phase-space coordinates of the centre of mass of the Sun's birth cluster $\left(\mathbf{x}_{\mathrm{cm}}, \mathbf{v}_{\mathrm{cm}}\right)$ are computed by integrating the orbit of the Sun backwards in time, taking into account the uncertainty in its current Galactocentric position and velocity. By using this technique, Martínez-Barbosa et al. (2015) found the distribution of possible positions and velocities of the Sun at its birth $\left(P\left(R_{\mathrm{b}}\right)\right.$, $\left.P\left(V_{\mathrm{b}}\right)\right)$. Given that for most of the Galactic parameters, $\left(P\left(R_{\mathrm{b}}\right)\right)$ is peaked around $9 \mathrm{kpc}$, we assume initially for the Sun's birth cluster $r_{\mathrm{cm}}=9 \mathrm{kpc}$. The Sun's birth cluster is then evolved under the influence of its self-gravity and the strong external tidal field produced by the Galaxy. We also consider stellar evolution effects. We used the HUAYNO code (Pelupessy et al.2012) to compute the internal gravitational force in the Sun's birth cluster. To compute the force due to the external Galactic potential, we use a high-order Rotating BRIDGE (Martínez-Barbosa et al. 2015). The stellar evolution effects were modelled with the SEBA code (Portegies Zwart \& Verbunt 1996, Toonen et al. 2012). These codes were coupled through the AMUSE framework (Portegies Zwart et al. 2013).

\section{Results}

Since the age of the Sun is approximately 4.6 Gyr (Bonanno et al.2002), we evolved the Sun's birth cluster over that period of time in the Galactic model described previously. We used different initial masses and radii and different combinations of bar and spiral arms parameters. At the end of each simulation, the Sun's birth cluster is completely disrupted, with the solar siblings located in different regions 

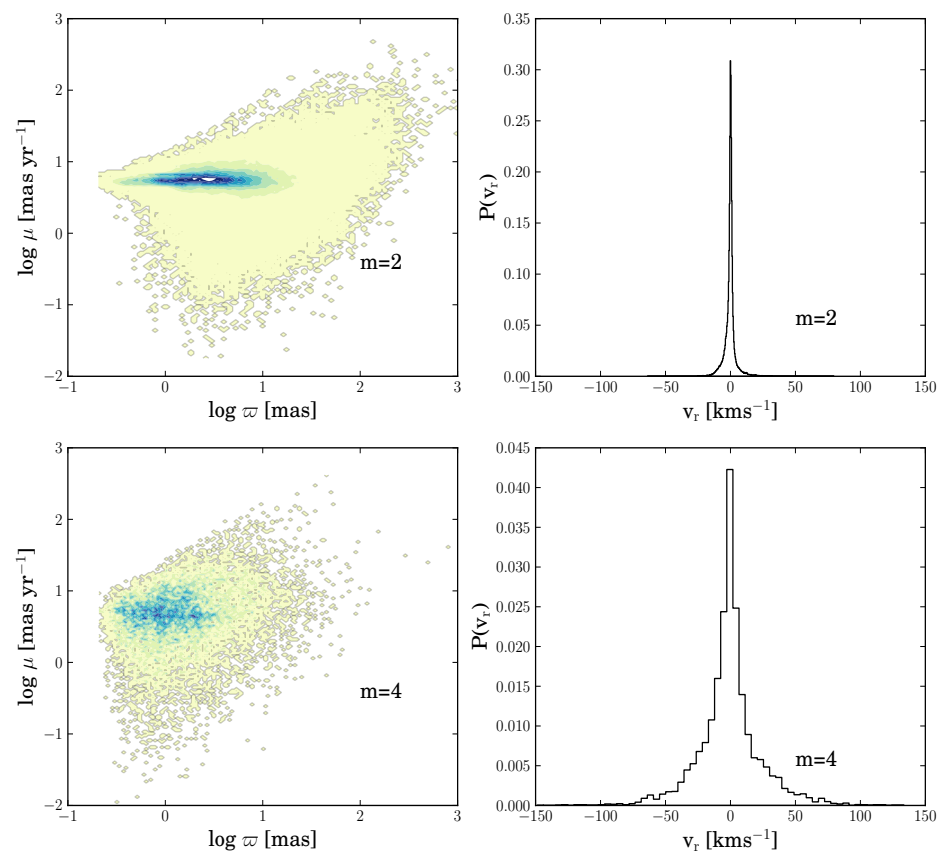

Fig. 1. Left: Parallax Vs. proper motion of the solar siblings. Right: distribution of radial velocity of the solar siblings. Top: Astrometric properties when the Galaxy has two spiral arms. Bottom: Astrometric properties when the Galaxy has four spiral arms. We varied the mass and pattern speed of the bar and the amplitude and pattern speed of the spiral arms.

of the Galactic disk. The radial and azimuthal dispersion of the final (present day) distribution of solar siblings depends on the combination of Galactic parameters. From the galactocentric distribution of solar siblings, we compute their parallaxes, proper motions and radial velocities. An example is shown in Fig. 1. Here we have superposed the astrometric properties of the solar siblings for 130 different combinations of bar and spiral arms parameters. The initial mass and radius of the Sun's birth cluster were fixed to $500 M_{\odot}$ and 0.5 parsec respectively. As can be observed, the solar siblings span a large range of parallaxes and proper motions (e.g. left panel Fig. 1). The dark blue region corresponds to the area in phase-space where most of the solar siblings are located. The parallax of the solar siblings in this region is in the range $0.3 \lesssim \varpi \lesssim 10$ mas; therefore, most of the solar siblings are relatively far from the Sun. Note that the proper motion of the solar siblings there is around 6 mas $\mathrm{yr}^{-1}$, which can be explained as stars moving along the solar circle with velocities comparable to that of the Sun (Brown et al. 2010). The distribution of radial velocities is centered around $0 \mathrm{kms}^{-1}$ (e.g. 
Title : will be set by the publisher

right panel of Fig. 1). The small relative motion of the solar siblings with respect to the Sun is due to the Galactic model used in the simulations. Non-transient spiral structure does not produce significant radial migration. Transient spiral arms and the interaction of the Sun's birth cluster with giant molecular clouds (GMCs) would produce a broader range of phase space coordinates and therefore broader radial velocity distributions.

Even though in the simulations the Sun's birth cluster is not much affected by radial migration, we found that the expected number of solar siblings predicted to be observed by Gaia is not higher than 100. If we assume four spiral arms in the Galactic model, this number is reduced to 50 stars on average. Given that the Gaia catalogue will contain one billion stars, it will be extremely difficult to find solar siblings using only phase space, even if we look for a contrast between the simulated phase-space of solar siblings and the disk stars of the Gaia data base (as done in Brown et al. (2010) ). Therefore, in order to find possible solar siblings, it is necessary to make use of the chemical tagging technique combined with the phase space selection criteria established through the simulations.

\section{References}

Allen, C., Santillán, A. 1991, Rev. Mex. Astron. Astrofis., 22, 255

Antoja, T., Figueras, F., Romero-Gómez, M., Pichardo, B., Valenzuela, O., Moreno, E. 2011, MNRAS, 418, 1423

Bonanno, A., Schlattl, H., \& Paternó, L. 2002, A\&A, 390, 1115

Brown, A. G. A., Portegies Zwart, S. F., Bean J. 2010, MNRAS, 407, 458

Ferrers, N. M. 1877, Pure Appl. Math., 14, 1

Lada, E. A., Strom, K. M., \& Myers, P. C. 1993, in Protostars and Planets III, ed. E. H. Levy \& J. I. Lunine (Tucson, AZ: Univ. Arizona Press), 245

Kroupa, P. 2001, MNRAS, 322, 231

Lindegren, L. et al. 2008, in Jin W. J., Platais I., Perryman M. A. C., eds, Proc. IAU Symp. 248, A Giant Step: From Milli- to Micro- Arcsecond Astrometry. Cambridge Univ. Press, Cambridge, p. 217

Looney, L. W., Tobin, J. J., \& Fields, B. D. 2006, ApJ, 652, 1755

Malhotra, R. 2008, in AAS/Division for Planetary Sciences Meeting Abstracts, No. 38.01, Vol. 40

Martínez-Barbosa, C.A., Brown, A.G.A., Portegies Zwart, S. 2015, MNRAS, 446, 823

Morbidelli, A., \& Levison, H. F. 2004, AJ, 128, 2564

Pelupessy, F. I., Jänes, J., Portegies Zwart, S. 2012, New Astronomy, 17, 711

Plummer, H. C. 1911, MNRAS, 71, 460

Portegies Zwart, S. F., Verbunt, F. 1996, A\&A, 309, 179

Portegies Zwart, S. F. 2009, ApJ, 696, L13

Portegies Zwart, S., McMillan, S. L. W., van Elteren, E., Pelupessy, I., de Vries, N. 2013, Computer Physics Communications, 183, 456

Toonen, S., Nelemans, G., Portegies Zwart, S., 2012, A\&A, 546, A70 